\title{
Peripheral Eosinophil Counts Correlate With Nasal Eosinophil Counts in Patients With Rhinitis
}

\author{
Ciprandi $\mathrm{G}^{1}$, Varricchio $\mathrm{A}^{2}$, Tajana $\mathrm{G}^{3}$, La Mantia $\mathrm{I}^{4}$, Tommasino $\mathrm{C}^{5}$ \\ ${ }^{1}$ Associazione Italiana Vie Aeree Superiori, Naples, Italy \\ ${ }^{2} U O S D$ di Video-Endoscopia delle VAS, P.O. San Gennaro - Asl \\ Napoli1-centro, Naples, Italy \\ ${ }^{3}$ Anatomy and Embriology, University of Salerno, Salerno, Italy \\ ${ }^{4}$ ENT Department, University of Catania, Catania, Italy \\ ${ }^{5}$ UO Patologia Clinica, P.O. San Gennaro - Asl Napoli1-centro, \\ Naples, Italy
}

J Investig Allergol Clin Immunol 2018; Vol. 28(6): 428-430

doi: 10.18176/jiaci.0306

Key words: Eosinophils. Nose. Blood. Cytometry. Rhinitis.

Palabras clave: Eosinófilos. Nariz. Sangre. Citometría. Rinitis.

Eosinophilic inflammation affecting the nose indicates a $\mathrm{T}_{\mathrm{H}} 2$ immune response, which is typical in allergic rhinitis and in nonallergic rhinitis with eosinophils (NARES), as well as in eosinophilic asthma [1]. Nasal cytology is a convenient method that is very useful in clinical practice, mainly in the diagnostic and prognostic work-up of patients with rhinitis [2]. In addition, it has been reported that the nasal eosinophil count correlates better with symptom severity and IgE level $[3,4]$.

In their cross-sectional study of adults with moderatesevere asthma, Amorim et al [5] demonstrated a convincing association between nasal and sputum eosinophilia and a link between the former and bronchodilator response, ie, postsalbutamol $\mathrm{FEV}_{1}$. These results agree with those of recent studies that showed close similarities in tissue inflammatory changes in asthma and rhinitis, further supporting the concept that the upper and the lower airways should be considered a single entity influenced by common physiologic processes, namely, the one-airway hypothesis [6]. Therefore, the evaluation of upper airway inflammation may provide additional insight into lower airway involvement and suggests that evaluation of nasal eosinophilia could be a surrogate for sputum analysis in these patients. In other words, nasal eosinophils may mirror bronchial eosinophils, thus enabling the nose to be considered the window of the bronchi.

Another pathway for indirect evaluation of bronchial eosinophils is through blood eosinophils. Peripheral eosinophils have been reported to be a reliable surrogate biomarker for phenotyping type 2 asthma [7].

Therefore, we tested the hypothesis of whether peripheral eosinophil count is correlated with nasal eosinophil counts. To verify this possibility, we compared nasal eosinophils with blood eosinophils in a group of patients with rhinitis in a realworld setting. The study sample comprised 41 consecutive patients (23 males, 18 females; mean age, 38.7 years) attending a rhinology clinic who were enrolled on 2 consecutive days. All patients underwent a through otorhinolaryngologic examination (including endoscopy, nasal scraping, and nasal 

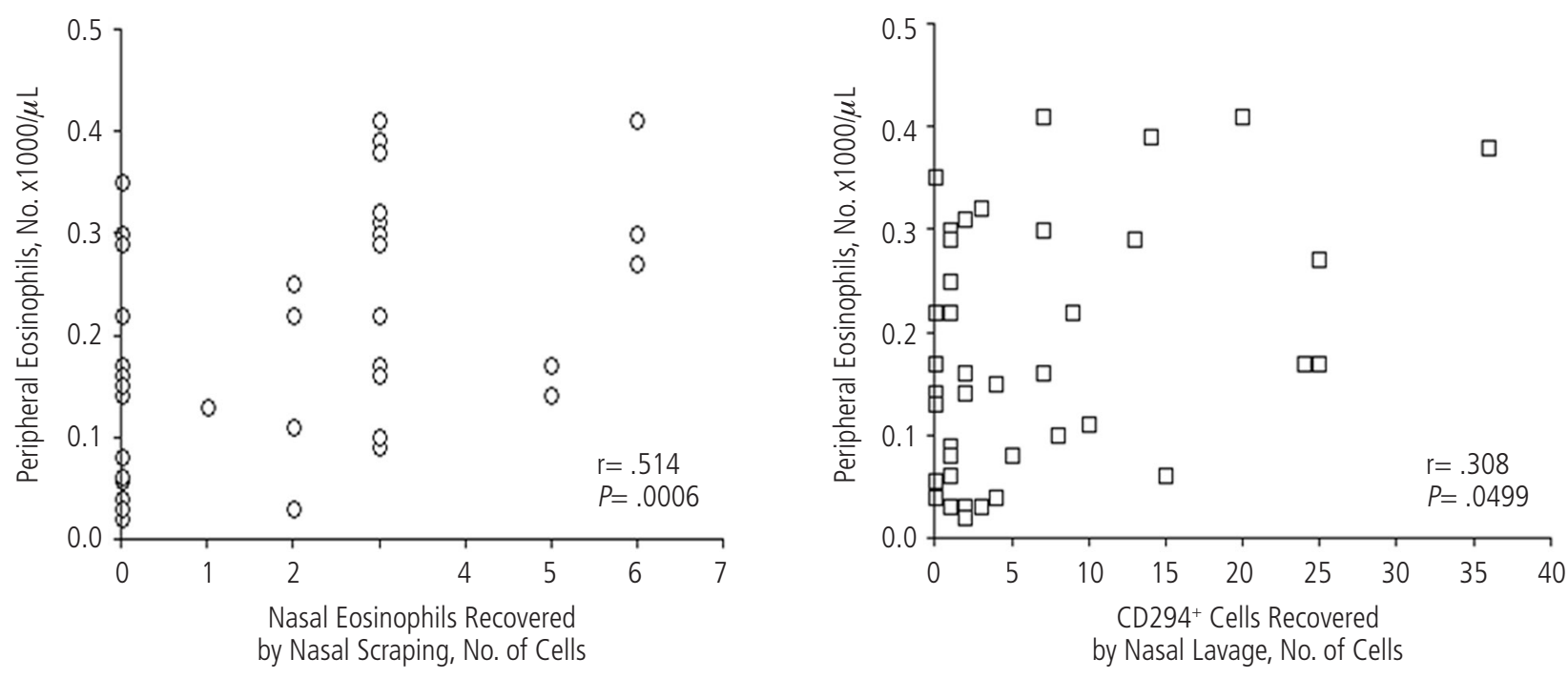

Figure. A, Relationship between peripheral eosinophils and nasal eosinophils recovered by nasal scraping (mean numbers of 10 fields; original magnification, $\times 1000$ ) and identified by microscopy. B, Relationship between peripheral eosinophils and CD294+CD203c cells recovered by nasal lavage and identified by flow cytometry.

lavage) and had inflammatory rhinitis (15 allergic rhinitis, 10 NARES, and 11 nonallergic noneosinophilic rhinitis). Diagnosis of allergic rhinitis was based on the agreement between a positive result in the skin prick test and the occurrence of nasal symptoms after exposure to the sensitizing allergen. The diagnosis of NARES was based on nasal eosinophilia ( $\geq 5 \%$ of cell count) and a negative allergy test result. The diagnosis of nonallergic noneosinophilic rhinitis was based on the presence of inflammatory cell infiltrate in the absence of allergy and a diagnosis of NARES.

Nasal scraping for traditional cytology was performed according to validated criteria [2]. Nasal lavage was performed by slowly instilling $10 \mathrm{~mL}$ of sterile isotonic saline into each nostril using a $10-\mathrm{mL}$ syringe while the patient reclined his/ her head and closed the soft palate. The solution was retained for approximately 10 seconds in the nasal cavities without swallowing. It was then expulsed by flexing the head forward, exhaling lightly, and rinsing lavage fluid into a sterile plastic beaker. Patients were strictly instructed to collect only secretions from the nose in the sterile beaker; secretions from the mouth had to be spat into the toilet. Immediately after collection, nasal lavage fluid was cytocentrifuged and the cell pellet was suspended in flow cytometry buffer (PBS, 0.09\% sodium azide, $1 \%$ heat-inactivated FBS) and stained with monoclonal antibodies to CD294 and CD203c (CD294 positivity and CD203c negativity identify eosinophils) for 20 minutes at room temperature in the dark. Cells were washed with flow cytometry buffer, resuspended in $0.5 \%$ paraformaldehyde, and stored at $4^{\circ} \mathrm{C}$ in the dark. Samples were acquired within 24 hours on a flow cytometer (Cytomics FC 500, Beckman Coulter Diagnostics). Isotype-matched single color controls were used to control for nonspecific staining and to set analysis gates.

As shown in the Figure, A, the relationship between peripheral blood eosinophils and nasal eosinophils, as evaluated using classic nasal cytology performed by scraping, was moderate $(\mathrm{r}=0.51 ; P<.001)$. The relationship between peripheral blood eosinophils and nasal CD294 ${ }^{+}$CD $203 \mathrm{c}^{-}$cells recovered from lavage fluid and assessed using flow cytometry, was weaker ( $\mathrm{r}=0.31 ; P<.05$; see Figure, $\mathrm{B})$. As expected, there was a strong relationship between nasal eosinophils recovered by nasal scraping and nasal CD294 ${ }^{+} \mathrm{CD} 203 \mathrm{c}^{-}$cells recovered by nasal lavage $(\mathrm{r}=0.65 ; P<.0001)$.

In a subanalysis of the 15 allergic patients, the relationship between nasal eosinophils (obtained by scraping) and blood eosinophils was strong $(\mathrm{r}=0.66 ; P<.01)$, whereas in nonallergic patients the relationship was weak $(\mathrm{r}=0.394)$. Finally, we calculated a cut-off peripheral eosinophil value to be able to predict the presence of a nasal eosinophilic infiltrate. The area under the ROC curve was 0.74 (95\%CI, 0.58-0.87) for a value of 80 eosinophils/ $\mu \mathrm{L}$ (sensitivity, 91.3\% [95\%CI, 71.9-98.7]; specificity, 55.6\% [95\%CI, 30.8-78.4]; positive likelihood ratio, 2.05; negative likelihood ratio, 0.16 ).

Therefore, this real-world study shows that peripheral blood eosinophils correlate well with the presence of nasal eosinophils in patients with nasal symptoms, as assessed by nasal scraping and microscopic observation. Nasal lavage and identification of eosinophils by flow cytometry correlated worse with blood eosinophils, although the correlation between both methods was very good. Of note, classic nasal cytology (scraping and microscopic identification) is a cheaper and more convenient test than nasal lavage and flow cytometry.

Nonetheless, our study has a series of limitations, including the limited number of patients, the cross-sectional design, and the lack of an assessment of symptom severity. Therefore, further studies should be conducted to corroborate our findings.

Our findings suggest that peripheral eosinophil count could be a potential biomarker for suggesting the presence of eosinophilic nasal infiltrate in clinical practice. Indeed, a value $>80 \mu \mathrm{L}$ could be considered suggestive of eosinophilic nasal inflammation when associated with a clinically relevant history. 
In conclusion, peripheral eosinophil count correlates well with nasal eosinophil count and could reasonably be considered a biomarker for suspecting type 2 inflammation in the nose.

Funding

The authors declare that no funding was received for the present study.

Conflicts of Interest

The authors declare that they have no conflicts of interest.

\section{References}

1. Roufosse F. Targeting the Interleukin-5 Pathway for Treatment of Eosinophilic Conditions Other than Asthma. Front Med. 2018:5:49

2. Gelardi M, Lannuzzi L, Quaranta N, Landi M, Passalacqua G. NASAL cytology: practical aspects and clinical relevance. Clin Exp Allergy. 2016;46:785-92.

3. Badorrek P, Müller M, Koch W, Hohlfeld JM, Krug N. Specificity and reproducibility of nasal biomarkers in patients with allergic rhinitis after allergen challenge chamber exposure. Ann Allergy Asthma Immunol 2017;118:290-7.

4. Burte E, Bousquet J, Siroux V, Just J, Jacquemin B, Nadif R. The sensitization pattern differs according to rhinitis and asthma multimorbidity in adults: the EGEA study. Clin Exp Allergy. 2017;47:520-9.

5. Amorim MM, Araruna A, Caetano LB, Cruz AC, Santoro LL, Fernandes LG. Nasal eosinophilia: an indicator of eosinophilic inflammation in asthma. Clin Exp Allergy 2010;40:867-74.

6. Alduraywish SA, Lodge CJ, Campbell B, Allen KJ, Erbas B, Lowe AJ, et al. The march from early life food sensitization to allergic disease: a systematic review and meta-analyses of birth cohort studies. Allergy. 2016;71:77-89.

7. Johansson MW. Eosinophil Activation Status in Separate Compartments and Association with Asthma. Front Med. 2017;4:75.

Manuscript received June 16, 2018; accepted for publication August 20, 2018.

Giorgio Ciprandi

Genoa, Italy

E-mail: gio.cip@libero.it 